\title{
NOUVELle
}

\section{Enfermé dehors}

Traitement de l'hépatite C chronique par un inhibiteur d'entrée

Laurent Mailly $^{1,2}$, Mirjam B. Zeisel ${ }^{1,2}$, Thomas F. Baumert ${ }^{1-3}$
${ }^{1}$ Inserm U1110, Institut de recherche sur les maladies virales et hépatiques, 3, rue Koeberlé, 67000 Strasbourg, France ;

${ }^{2}$ Université de Strasbourg, rue Blaise Pascal, 67000

Strasbourg, France;

${ }^{3}$ Institut hospitalo-universitaire (IHU), Pôle hépato-digestif, Hôpitaux universitaire de Strasbourg, 67000 Strasbourg, France.

laurent.mailly@unistra.fr

\section{L'infection chronique par le virus} de l'hépatite C : quelles options thérapeutiques?

Le virus de l'hépatite C (VHC) est une cause majeure d'hépatite chronique, pouvant évoluer en cirrhose hépatique puis en carcinome hépatocellulaire [1]. Environ 170 millions de personnes sont porteuses de ce virus dans le monde et, pour l'heure, aucun vaccin n'est disponible. Plusieurs molécules ciblant directement le virus, appelées antiviraux à action directe, ont récemment été mises sur le marché. Ces antiviraux ont révolutionné la prise en charge des patients atteints d'hépatite $C$ chronique, faisant passer le taux de guérison d'environ $50 \%$ à environ $90 \%$. Néanmoins, le coût de ces traitements reste très élevé et ceuxci ne sont accessibles qu'à une minorité de patients. De plus, ces traitements n'ont pas la même efficacité contre tous les génotypes de virus, ni chez tous les patients. Les patients avec les atteintes hépatiques les plus graves nécessitent en effet des traitements plus complexes et, pour certains, une transplantation hépatique. Cependant, le greffon est systématiquement réinfecté par le virus, entraînant une accélération du développement de la maladie hépatique. Le potentiel des antiviraux à action directe dans le traitement des patients infectés par le VHC avant/après la transplantation hépatique est actuellement en cours d'études cliniques ${ }^{1}$. De nouvelles options préventives et thérapeutiques sont également envisagées afin d'améliorer la

${ }^{1}$ Plusieurs essais cliniques sont en cours et peuvent être retrouvés sur clinicaltrials.gov prise en charge des patients et d'aboutir à une éradication de l'infection.

L'entrée du virus de l'hépatite C : un processus complexe

L'entrée du virus dans sa cellule hôte est une des étapes clé pour l'installation de l'infection mais également pour son maintien et pour la dissémination du virus. Cette étape cruciale fait intervenir plusieurs facteurs cellulaires dont les protéoglycanes à héparanes sulfates ${ }^{2}$ (HSPG), le récepteur des lipoprotéines de faible densité ( $L D L-R$ ), le récepteur scavenger de classe $B$ type $\mathrm{I}^{3}(\mathrm{SR}-\mathrm{BI})$, les tétraspanines ${ }^{4} \operatorname{CD} 81$ et $C D 63$, le récepteur du facteur de croissance épidermique $^{5}$ ( $\left.\varepsilon G F R\right)$, le récepteur de l'éphrine $A 2^{6}$ (EphA2), le récepteur de la transferrine $^{7}$ (TfRl), le facteur Niemann-Pick Cl like $1^{8}$ (NPClLl) et les protéines de jonctions serrées, occludine ${ }^{9}$ (OCLN) et claudine-1 ${ }^{10}$ (CLDNl) [2]. Ce mécanisme est progressif dans le temps et dans l'espace. En effet, le VHC circulant, associé à des lipoprotéines, interagirait en premier

\footnotetext{
2 Protéine de la matrice extracellulaire.
}

${ }^{3}$ Récepteur reconnaissant notamment les lipoprotéines.

${ }^{4}$ Protéines transmembranaires participant à la régulation de l'activité des cellules immunitaires.

${ }^{5}$ Le facteur de croissance épidermique EGF est une hormone agissant dans de nombreux tissus (pas uniquement épidermique) impliquée dans le contrôle de l'expression génétique.

${ }^{6}$ La voie de signalisation Eph/ephrin participe à de nombreux mécanismes embryonnaires mais aussi, à l'âge adulte, à l'angiogenèse, la différenciation cellulaire, et les interactions entre cellules.

${ }^{7}$ La transferrine est une protéine sérique synthétisée par le foie et participant au transport du fer

${ }^{8}$ Facteur impliqué, au niveau des hépatocytes, dans la réabsorption du cholestérol.

${ }^{9}$ Protéine transmembranaire impliquée dans la cohésion des jonctions serrées.

10 Protéine transmembranaire, élément principal des jonctions serrées. lieu avec les HSPG, puis le LDL-R et le $S R-B I$, ce qui permettrait de concentrer le virus à la surface de la cellule. S'en suivraient alors des mouvements de protéines à la surface cellulaire et une interaction directe, ou indirecte, du virus avec les autres facteurs, notamment le complexe CD81-CLDN1 indispensable à l'entrée virale [3]. L'association de ces deux facteurs serait contrôlée, lors de l'infection, par des enzymes intracellulaires activées par l'EGFR, notamment la petite protéine $\mathrm{G} \mathrm{H}$-Ras [4]. Le rôle des autres facteurs, tels que NPCILl, TfRl et OCLN, semble moins clair car aucune interaction directe du virus avec ces protéines à la surface cellulaire n'a été montrée à ce jour. Le mécanisme d'entrée complexe du VHC offre donc différentes cibles qui peuvent toutes devenir des options thérapeutiques prometteuses si tant est qu'il soit possible de les cibler spécifiquement.

\section{Utilisation des inhibiteurs d'entrée} pour la prévention de l'infection par le virus de l'hépatite $\mathrm{C}$

L'entrée virale constitue la première étape du cycle viral et joue un rôle clé dans la pathogenèse de l'infection du greffon au cours de la transplantation hépatique. Des inhibiteurs d'entrée pourraient donc être particulièrement intéressants pour prévenir l'infection virale dans le cadre de la transplantation hépatique. Certaines études réalisées in vivo utilisant un modèle de souris chimériques au foie humanisé pouvant être infectées par le VHC, ont déjà montré la possibilité de prévenir ou de retarder l'infection en ciblant différents facteurs d'entrée par de 


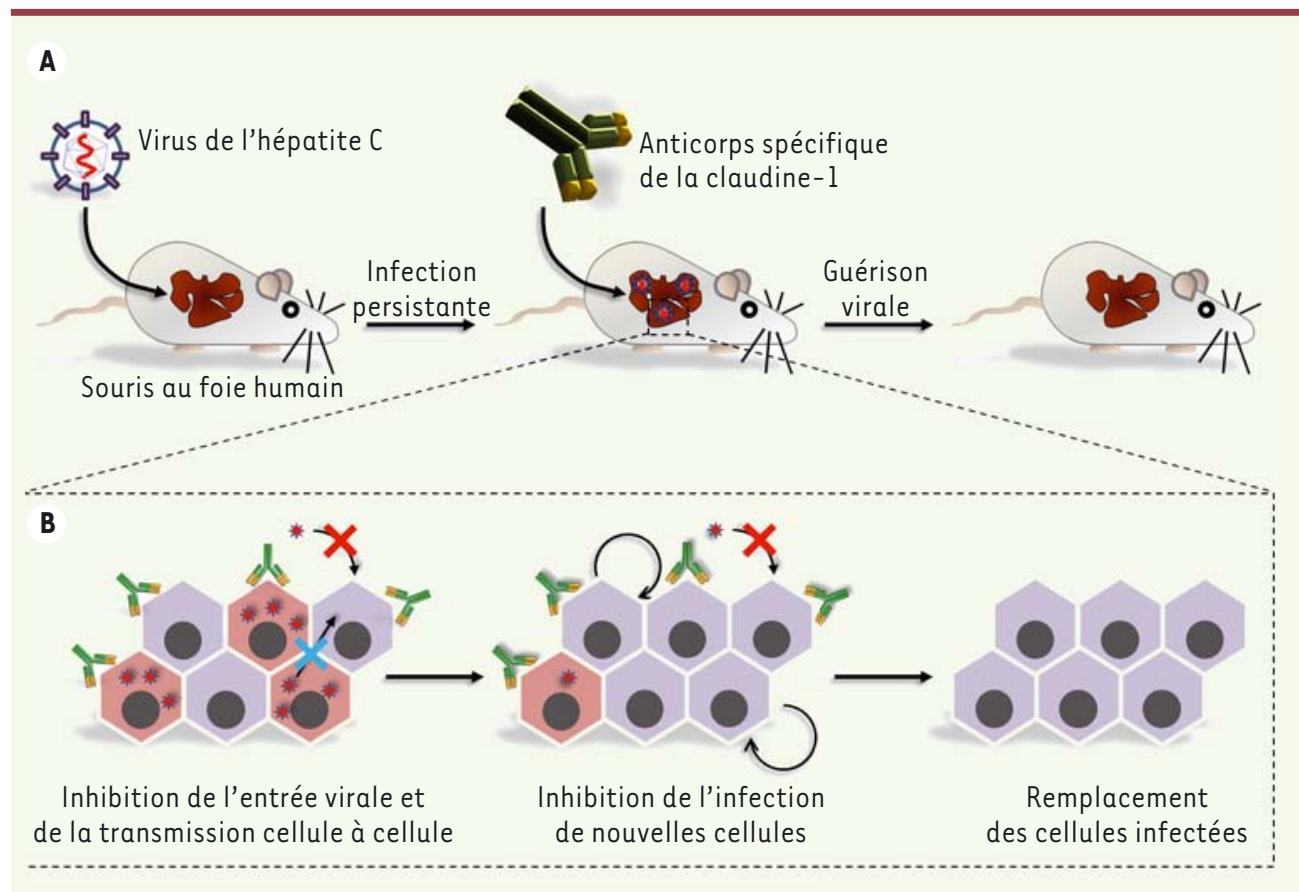

Figure 1. Mécanisme d'action de l'anticorps anti-claudine-1 (CLDN1) conduisant à l'éradication du VHC in vivo. A. Les souris UPA-SCID (urokinase type plasminogen activator - severe combined immunodeficiency), au foie chimérique humanisé, peuvent être efficacement infectées de façon persistante par le virus de l'hépatite C (VHC). Ce modèle a été utilisé pour étudier l'efficacité de l'anticorps anti-claudine-1 (CLDNI) pour traiter une infection persistante par le VHC. Une fois l'infection établie, les souris sont traitées par une injection hebdomadaire d'anticorps anti-CLDNl pendant 4

semaines. La charge virale détectée dans le sérum permet de vérifier l'élimination du virus. B. Au niveau cellulaire, l'anticorps inhibe l'entrée de novo du virus dans les cellules non infectées (croix rouge) ainsi que la transmission de cellule à cellule (croix bleue). Les cellules non infectées peuvent ainsi proliférer (flèche circulaire) pour remplacer les cellules infectées qui meurent par apoptose induite par le virus (modifié à partir de [16]).

petites molécules. L'inhibition de l'EGFR par l'erlotinib, une molécule approuvée pour le traitement du cancer du poumon, ralentit et diminue l'infection par le VHC dans ces souris [5]. De la même façon, l'inhibition du NPClLl par l'ézétimibe, une molécule utilisée pour le traitement de l'hypercholestérolémie, permet de prévenir l'infection dans certaines souris chimériques [6]. Ces études montrent le potentiel de réorientation de médicaments, déjà sur le marché et approuvés pour d'autres pathologies, pour la prise en charge de l'hépatite C. L'infection par le VHC peut également être prévenue in vivo, en ciblant le CD81 ou le SR-BI par l'intermédiaire d'anticorps monoclonaux $[7,8]$. II est à noter qu'il est possible d'inhiber l'infection en injectant un anticorps anti-SR-BI six heures après l'inoculation du virus [9], ce qui suggère que la fenêtre de temps pour l'administration d'un inhibiteur d'entrée, pour prévenir l'infection, n'est pas limitée à la période pré-infection. Les mécanismes de l'entrée virale jouant également un rôle dans le maintien de l'infection chronique par le VHC, l'utilisation d'inhibiteurs d'entrée pourrait s'avérer efficace pour éradiquer le virus après une infection déjà établie depuis plusieurs semaines. Aucune étude ne s'était intéressée à cette possibilité jusqu'à présent.

\section{Claudine-1 : une cible pour}

la prévention et le traitement de l'infection par le virus de l'hépatite C Notre laboratoire a produit et caractérisé différents anticorps monoclonaux dirigés contre la claudine-1 (CLDN1), et l'un d'entre eux, le clone 0M-7D3-B3, s'est montré particulièrement efficace in vitro pour inhiber l'entrée du VHC [10]. Nous avons utilisé le modèle de souris chimériques afin de tester l'efficacité de cet anticorps anti-CLDNl in vivo [11]. Contrairement aux études précédentes, nous avons évalué l'efficacité de l'anticorps à la fois dans la prévention de l'infection par le VHC et dans le traitement d'une infection persistante, en administrant l'anticorps avant, pendant ou après l'inoculation du virus. De manière intéressante, nous avons clairement démontré que cet anticorps pouvait non seulement prévenir, mais également traiter, une infection par le VHC in vivo. Des souris infectées depuis 4 à 8 semaines ont en effet vu leur charge virale chuter très rapidement, en 2 à 4 semaines, sous le seuil de quantification jusqu'à devenir indétectable. La guérison virologique, correspondant à l'élimination du virus de l'organisme, s'est montrée durable chez la grande majorité des souris jusqu'à au moins 12 semaines après le début du traitement, et ce, sans développement de résistance virale. De plus, différents tests in vivo, ex vivo et in vitro n'ont révélé aucun effet toxique de l'anticorps utilisé. Cette absence de toxicité est très probablement due au fait que l'anticorps ne se fixe pas sur la CLDNl engagée dans la formation de jonctions serrées, mais plutôt sur la CLDNl exprimée sur la membrane baso-latérale des hépatocytes. Des tests in vivo, 
ex vivo et in vitro montrent en effet que l'anticorps n'induit pas de rupture des jonctions serrées. II n'interagirait donc pas avec les fonctions physiologiques de la CLDN1. D'un point de vue mécanistique, cet anticorps empêche l'association de la CLDNl avec le CD81, étape cruciale pour l'entrée virale [3], et inhibe la transmission virale de cellule à cellule, et donc, la dissémination du virus. Il se montre également capable de contrecarrer l'activation de voies de signalisations intracellulaires qui interviennent lors de l'infection, notamment la phosphorylation des protéines ERK1/2 (extracellular signal-regulated protein kinases 1/2). In situ, l'anticorps conduit à l'éradication des cellules infectées. Le renouvellement des cellules non infectées et protégées de l'infection par l'anticorps, permet en effet un remplacement progressif des cellules infectées, celles-ci mourant vraisemblablement d'apoptose induite par le virus (Figure 1). Nous avons réalisé une modélisation mathématique de la diminution de la charge virale suite au traitement avec l'anticorps afin de tester l'hypothèse selon laquelle cet anticorps interfère uniquement avec l'étape d'entrée virale et non avec plusieurs étapes du cycle du virus incluant sa réplication. Nos résultats indiquent que chez les souris, la demi-vie des cellules infectées se situerait autour des 1,3 jours pour le VHC de génotype $2 a$ et 5,4 jours pour le VHC de génotype 4 , ce qui correspond à ce qui a été décrit chez des patients chroniquement infectés, validant ainsi l'utilisation du modèle murin pour ces expériences [12]. Cibler une molécule de l'hôte, en l'occurrence un facteur d'entrée indispensable à l'établissement et au maintien de l'infection, semble donc être une stratégie thérapeutique très prometteuse. Au cours de cette étude, l'anticorps a été administré en monothérapie. Les traitements actuels contre l'hépatite $C$ requièrent, eux, une association de plusieurs molécules afin de minimiser les risques de résistance. De manière intéressante, nous avons montré in vitro que l'anticorps anti-CLDNl pouvait être associé à des antiviraux à action directe, et ainsi potentialiser l'efficacité de ces antiviraux tout en permettant de diminuer les doses des différents composés afin d'en réduire les effets secondaires [13].

\section{Les protéines de jonctions serrées :} une cible antimicrobienne universelle? De nombreux agents pathogènes pénètrent dans leur cellule hôte via des protéines de jonctions serrées. Parmi ceux-ci, nous pouvons mentionner, par exemple, le virus de la dengue qui interagit également avec la CLDN1, certains adénovirus et coxsackievirus ${ }^{11}$ qui utilisent la molécule CAR (coxsackie and adenovirus receptor), les shigelles ${ }^{12}$ qui entrent dans la cellule hôte via la tricelluline ${ }^{13}$, et le réovirus ${ }^{14}$ qui se fixe à la protéine JAM-A (junctional adhesion molecule-A) [14]. Ces protéines de jonctions serrées, agissant comme portes d'entrée universelles pour de nombreux microorganismes, représentent donc des cibles potentielles pour la prévention et le traitement de ces infections. La mise au point d'anticorps, ou de petites molécules, ciblant ces protéines cellulaires peut ainsi être envisagée comme une approche antimicrobienne pour un grand nombre de pathogènes. L'inhibition des mécanismes d'entrée dans la cellule hôte permet de fermer la porte aux pathogènes afin d'éviter une infection persistante et leur propagation illimitée. En combinaison avec d'autres approches thérapeutiques, cette stratégie peut notamment se montrer intéressante afin de faciliter l'éradication de pathogènes s'installant de façon chronique $[13,15]$. $\diamond$

\footnotetext{
${ }^{11}$ Les virus à Coxsackie sont des virus non enveloppés à ARN simple brin. Ils sont responsables de nombreuses pathologies comme la maladie pieds-mains-bouche, l'hépatite, ou certaines méningites.

${ }^{12}$ Bactéries du genre Shigella, pathogènes et spécifiques du tube digestif.

13 Protéine des jonctions serrées au niveau des contacts entre trois cellules.

${ }^{14}$ Famille de virus à ARN à double brin, affectant particulièrement le système digestif ou le système respiratoire.
}

Locked outside: cure of chronic hepatitis $C$ using an entry inhibitor

\section{LIENS D'INTÉRÊT}

L'Inserm, l'Université de Strasbourg et Genovac/ Aldevron Freiburg ont déposé une demande de brevet pour l'anticorps monoclonal anti-claudine 1 pour l'inhibition de l'infection par le virus de l'hépatite C (US patent \# 8518408; W02010034812; PCT/ EP2009/062449). T.F.B. a servi de conseiller scientifique pour Gilead, Biotest et Vironexx.

\section{RÉFÉRENCES}

1. Thomas DL. Global control of hepatitis C: where challenge meets opportunity. Nat Med 2013; $19: 850-8$.

2. Zona L, Turek M, Baumert TF, Zeisel MB. Hepatitis C virus internalization. Virologie $2013 ; 17$ : 401-13

3. Harris HJ, Farquhar MJ, Mee CJ, et al. CD81 and claudin 1 coreceptor association: role in hepatitis $C$ virus entry. J Virol 2008 ; 82 : 5007-20.

4. Zona L, Lupberger J, Sidahmed-Adrar N, et al. HRas signal transduction promotes hepatitis $C$ virus cell entry by triggering assembly of the host tetraspanin receptor complex. Cell Host Microbe 2013 ; 13 : 302-13.

5. Lupberger J, Zeisel MB, Xiao F, et al. EGFR and EphA2 are host factors for hepatitis $C$ virus entry and possible targets for antiviral therapy. Nat Med 2011 ; 17 : 589-95.

6. Sainz B, Barretto N, Martin DN, et al. Identification of the Niemann-Pick Cl-like 1 cholesterol absorption receptor as a new hepatitis $\mathrm{C}$ virus entry factor. Nat Med 2012 ; 18 : 281-5.

7. Meuleman P, Hesselgesser J, Paulson M, et al. AntiCD81 antibodies can prevent a hepatitis $C$ virus infection in vivo. Hepatology 2008 ; 48 : 1761-8.

8. Lacek K, Vercauteren K, Grzyb K, et al. Novel human $S R-B I$ antibodies prevent infection and dissemination of HCV in vitro and in humanized mice. J Hepatol $2012 ; 57: 17-23$

9. Vercauteren K, Van Den Eede N, Mesalam AA, et al. Successful anti-scavenger receptor class $B$ type $I$ (SR-BI) monoclonal antibody therapy in humanized mice after challenge with $\mathrm{HCV}$ variants with in vitro resistance to SR-BI-targeting agents. Hepatology $2014 ; 60: 1508-18$.

10. Fofana I, Krieger SE, Grunert F, et al. Monoclonal anti-claudin 1 antibodies prevent hepatitis $C$ virus infection of primary human hepatocytes. Gastroenterology 2010 ; 139 : 953-64.e4.

11. Mailly L, Xiao F, Lupberger J, et al. Clearance of persistent hepatitis $C$ virus infection in humanized mice using a claudin-1-targeting monoclonal antibody. Nat Biotechnol 2015 ; 33 : 549-54.

12. Neumann AU, Lam NP, Dahari H, et al. Hepatitis C viral dynamics in vivo and the antiviral efficacy of interferon-alpha therapy. Science 1998 ; 282 : 103-7.

13. Xiao F, Fofana I, Thumann C, et al. Synergy of entry inhibitors with direct-acting antivirals uncovers novel combinations for prevention and treatment of hepatitis C. Gut 2015; $64:$ 483-94.

14. Lu RY, Yang WX, Hu YJ. The role of epithelial tight junctions involved in pathogen infections. Mol Biol Rep 2014 ; 41 : 6591-610.

15. Uprichard SL, Sainz B, Jr. Inhibition of hepatitis C entry: too soon to dismiss while many are still being denied treatment. Gut $2015 ; 64: 690-1$.

16. Ray K. Hepatitis: Tightening the grip on clearing HCV infection. Nat Rev Gastroenterol Hepatol $2015 ; 12$ : 249 